Meta

Journal des tradlucteurs

Translators' Journal

\title{
Traduction signalétique
}

\section{Daniel Gouadec}

Volume 35, numéro 2, juin 1990

URI : https://id.erudit.org/iderudit/002945ar

DOI : https://doi.org/10.7202/002945ar

Aller au sommaire du numéro

Éditeur(s)

Les Presses de l'Université de Montréal

\section{ISSN}

0026-0452 (imprimé)

1492-1421 (numérique)

Découvrir la revue

Citer cet article

Gouadec, D. (1990). Traduction signalétique. Meta, 35(2), 332-341.

https://doi.org/10.7202/002945ar d'utilisation que vous pouvez consulter en ligne.

https://apropos.erudit.org/fr/usagers/politique-dutilisation/ 


\section{TRADUCTION SIGNALÉTIQUE}

DANIEL GOUADEC

Université Rennes 2, Rennes, France

\section{INTRODUCTION}

La traduction de textes dits pragmatiques (textes visant essentiellement à communiquer une information destinée à une exploitation spécifique) est un acte complexe, sollicitant de multiples activités interdépendantes et exigeant, par voie de conséquence, des compétences diverses.

Les textes pragmatiques peuvent se classer en catégories. Le classement peut intervenir par types: lettre/rapport/mode d'emploi/devis/facture/manuel/communication/ note/etc. Il peut également intervenir par domaines: textes commerciaux, textes d'informatique, textes de chimie, etc. Les classements importent, en définitive, peu: tous ces textes présentent deux caractéristiques liées fondamentalement:

a) ils sont «adaptés» à un public spécifique - y compris lorsqu'ils sont dits de vulgarisation;

b) ils sont destinés à une exploitation spécifique : vendre; obtenir paiement d'une facture; monter ou utiliser un appareil; ... ou, plus simplement, informer.

Les types de textes considérés seront diversifiés mais l'assujettissement à la spécificité d'une double destination (destination-public ET destination-exploitation) constituera une caractéristique constante.

La traduction des textes pragmatiques engage, dans sa complexité d'acte construit et raisonné, des activités complémentaires qui sont:

- une activité de compréhension préalable du texte à traduire,

- une activité d'interprétation préalable du texte à traduire,

une activité de transfert,

une activité de rédaction du texte résultant des transferts,

- une activité de révision ou de relecture.

Ces activités s'imbriquent (chronologiquement) les unes dans les autres et intègrent des activités annexes qui sont des activités de documentation ou de terminologie-terminographie, ou encore d'acquisitions spécialisées.

La diversité des apprentissages nécessaires à la maîtrise de l'acte de traduction explique et justifie la progression adoptée. Cette progression repose sur le principe d'un emboîtement de types de traductions dont chacun constitue la condition nécessaire du passage au type suivant. Elle repose parallèlement sur des observations ayant permis d'établir la nature des facteurs de risques présents en tel point du déroulement des processus de la traduction. Elle vise essentiellement à prévenir les distorsions en proposant des stratégies efficaces de la traduction.

Il semble indispensable, avant d'aborder la réflexion sur la pratique de la traduction, de s'interroger sur les causes majeures de dérapage. Nous ne citerons ici que les plus évidentes et les plus insidieuses.

1. la majorité des fautes de traduction sont, à l'évidence, des fautes de compréhension et d'interprétation du texte (compréhension du contenu brut du messagetexte et interprétation des finalités du texte ou des modalités qui lui ont donné forme). 
2. traduire, c'est construire un acte de communication efficace et non trouver des mots justes : il faut trouver le texte juste et, par conséquent, toujours contrôler pleinement un acte de rédaction un peu particulier.

3. traduire, c'est connaître les critères de traduction autrement qu'au moyen d'un corrigé type qui permet seulement de mesurer un écart entre sa propre performance et celle d'un maitre; c'est donc comprendre les stratégies, les enjeux, les objectifs d'un acte construit.

4. la traduction n'est pas une et indivisible: il existe plusieurs types de traductions adaptés, individuellement, à des situations de transfert ou truchement spécifiques.

5. l'erreur de traduction provient souvent d'une incapacité à définir ses objectifs: la traduction n'est pas un exercice universitaire (du moins dans la perspective que nous retenons).

6. prendre comme unité de travail des unités plus petites que le texte, c'est se condamner à l'erreur.

7. la traduction est trop souvent, dans les stades initiaux de la formation, traduction défensive ou traduction par calque corrigé seulement dans les cas où les ruptures par rapport à la logique du français deviennent flagrantes. Or, la traduction doit être offensive en ce sens que la maîtrise du texte français appartient au traducteur.

8. la pratique de la traduction tend à reposer, au départ, sur deux mythes liés - et fort dangereux :

E le mythe de l'omniscience du traducteur;

- le mythe de l'obligation de traduction absolue.

Le premier mythe veut que le traducteur soit capable de tout traduire parce qu'il connaît tout. Or, le traducteur consacre au moins la moitié de son temps de travail à APPRENDRE POUR COMPRENDRE.

Le second mythe veut que le traducteur soit capable de tout traduire parce que le blocage est signe d'une impardonnable carence. Or, tout traducteur professionnel sait que l'aveu d'ignorance ou d'impuissance vaut toujours mieux que le risque d'erreur par outrecuidance.

Les causes majeures d'erreurs de traduction résident dans: une absence de définition claire des objectifs et critères de la traduction; un déplacement des fonctions de la traduction; un découpage défectueux des unités de travail; un asservissement exagéré à l'original trop souvent «corrigé» par une «liberté abusive»; une incidence prématurée de la traduction proprement dite; des carences de compréhension de textes; une absence ou carence de savoirs spécifiques relatifs à l'objet du texte; un dépassement des compétences du traducteur; une inversion des séquences de progression de l'acte de traduction faisant de la mise en français un moyen de la compréhension et de la fidélité à l'organisation superficielle du texte une garantie de sérieux.

QUELQUES RÈGLES D'OR :

1. Apprendre pour comprendre

2. Différer au maximum la traduction

3. Tout comprendre avant de traduire

4. Ne jamais accepter une solution qui n'ait été confirmée

5. Adapter sa traduction aux besoins de l'utilisateur

6. Se souvenir toujours que la traduction engage (ou doit engager) une «équipe» dans laquelle le traducteur «collabore» avec: 

a. le donneur d'ouvrage
b. le technicien
c. le réviseur

et, le cas échéant, avec d'autres traducteurs.

7. En situation d'apprentissage :

- justifier ses solutions,

- proposer des hypothèses complémentaires,

- faire réviser ses travaux.

Lorsque l'on aborde la traduction des textes pragmatiques dans une optique préprofessionnelle, il importe de se pénétrer des quelques principes ci-dessous :

A. Une traduction juste en l'absence de compréhension suffisante du texte à traduire ne peut être que l'effet du hasard:

TRADUIRE, C'EST D'ABORD COMPRENDRE: PAS COMPRIS = PAS TRADUIT.

B. Le traducteur n'est pas omniscient, il est un apprenant perpétuel.

C. Nul ne peut traduire avec des chances raisonnables de réussite s'il ne sait réellement POUR QUI (pour quel public)

et POUR QUOI (pour quelle utilisation de son texte) il assure une médiation.

D. La traduction professionnelle impose des critères de qualité totalement différents de ceux qu'impose la traduction académique.

E. La traduction professionnelle, c'est d'abord une question de gestion des temps de travail. En effet, les détails sont toujours réduits eu égard à la complexité des activités que doit conduire le traducteur. Il importe donc d'aller vite sans que la vitesse d'exécution puisse nuire à la qualité.

F. Le traducteur professionnel est, généralement, le partenaire d'un réviseur ou relecteur (traducteur chevronné assurant une vérification et d'éventuelles corrections). Tous deux sont conjointement responsables de leur traduction commune. La présence du réviseur doit être systématiquement prise en compte.

G. Le traducteur n'est nullement assimilable à un prestidigitateur qui sortirait de son chapeau des solutions élégantes à tout problème posé: il enfante souvent dans l'énervement, le bruit, la douleur...

H. Le traducteur est, avant tout, maittre de la construction des textes : qui ne sait écrire ne sait traduire.

LES TRADUCTIONS : TYPES ET FONCTIONS

Les types de traductions - définis selon les destinations - s'organisent en une pyramide fondant une progression raisonnée des apprentissages.

\section{Types :}

1. Traduction SIGNALÉTIQUE

Nature:- recensement, en français, des déterminants du texte anglais.

Fonction : - indexation du texte permettant de déterminer si celui-ci porte une information utile ou pertinente (et, si tel est le cas, de déterminer le type de traduction complémentaire voulu).

\section{Traduction SÉLECTIVE/DOCUMENTAIRE}

Nature : - traduction exclusive des données relatives à un objet du texte autre que l'élément dominant dont il traite. 
Fonction : - accroître la vitesse d'accès à l'information par élimination de toute donnée non pertinente compte tenu de l'objet de documentation.

\section{Traductions SYNOPTIQUES}

a synoptique-source

- synoptique-analytique

aynoptique-contenu

Nature: - traductions condensées de la totalité des informations contenues dans un texte

- traduction des thèmes génériques du texte

description des contenus génériques + des finalités du texte et de ses sous-unités + des modalités de construction du texte.

- traduction abrégée de tous les contenus utiles d'un texte.

Fonction : - accès accéléré à des types d'information spécifiques.

\section{Traduction DIAGRAMMATIQUE}

Nature : - transfert, sous forme de diagramme, des données portées par le texte anglais.

Fonction : - a surtout une fonction essentielle du point de vue de la maitrise des stratégies standard du transfert par traduction.

\section{Traductions AVEC RECONSTRUCTIONS \\ analisée totale \\ - post-synthétique}

Nature : - transfert de la totalité des éléments d'un texte sans prise en compte absolue des accidents stylistiques.

u transfert de la totalité du texte avec adaptation à la nouvelle destination.

Fonction : - communiquer toute l'information sous la forme la plus simple.

- rendre l'information pleinement accessible ET obtenir un effet spécifique.

6. Traductions ABSOLUES

Nature : - transfert total respectant l'ensemble des critères de QUANTITÉ d'information et de QUALITÉ de communication.

7. TRADUCTION À VUE = transfert accéléré d'un texte ou document écrit (s'oppose aux traductions «à loisir» des types 1 à 6 ci-dessus). A surtout une fonction de renforcement des apprentissages.

Les divers types et sous-types de traductions répertoriés ci-dessus répondent à des critères de communication différents. Ils remplissent, dans la progression adoptée, une double fonction: une fonction d'étages de tri dans le cadre d'une pratique professionnelle; une fonction d'étages de test dans le cadre de la formation des traducteurs.

\section{ÉTAGES-TRI}

Les étages de tri se justifient, dans la pratique professionnelle, si l'on considère que la traduction absolue constitue parfois une traduction excessive. En effet, un utilisateur de traductions peut avoir des raisons variées de souhaiter un accès à l'information :

1. il peut simplement désirer savoir si tel texte contient une information qui lui serait utile. Dans ce cas, il lui suffirait d'une traduction signalétique. S'il s'avère qu'il y a effectivement information utile, une traduction de plus haut niveau sera entreprise ;

2. il peut, sachant que le texte est pertinent, ne pas désirer obtenir toute l'information. Dans ce cas, une traduction sélective suffit; 
3. il peut souhaiter toute l'information portée par le texte mais n'attacher aucune importance aux qualités proprement textuelles. Dans ce cas, une traduction diagrammatique ou une traduction synoptique-source fera l'affaire;

4. il peut souhaiter effectuer un classement de textes ou documents selon un système spécifique d'indexation. Dans ce cas, une traduction signalétique ou, mieux, une traduction analytique suffira;

5. il peut souhaiter savoir en gros de quoi traite le texte ou document et connaître, toujours en gros, les contenus. Dans ce cas, il serait sage de faire appel aux traductions synoptiques ou, peut-être, aux traductions banalisées;

6. il peut enfin souhaiter tout savoir et, dans ce cas, bien évidemment, il faudra lui fournir une traduction absolue.

Les étages-tri reposent sur une progressivité des formes de communication d'informations textuelles. À chaque étage, le client peut demander une traduction différente ou plus complète. Le travail du traducteur ne sera cependant jamais perdu puisque la pratique raisonnée OBLIGE, de toute façon, à passer par les différentes étapes ci-dessus (avec des variantes). L'objectif est simplement de ne pas perdre son temps en dépassant le niveau d'exigences correspondant aux besoins de l'utilisateur.

\section{ÉTAGES-TEST}

Les étages de test se justifient, dans la pratique didactique, si l'on considère que la traduction absolue n'est pas abordable d'emblée. Il semble donc raisonnable de vouloir établir une progression par niveaux homogènes dans laquelle chacun des niveaux définis correspondrait à un PRODUIT-TRADUCTION trouvant aussi une justification professionnelle.

Les étages-test interviennent au fil d'une progression qui comprendra, dans l'ordre :

1. la traduction signalétique

2. la traduction sélective

3. la traduction synoptique

4. la traduction diagrammatique

5. la traduction banalisée

6. la traduction analytique de second niveau

7. la traduction post-synthétique

8. la traduction absolue (deux sous-types)

et, de manière moins rigide, la traduction à vue.

Ainsi qu'on le notera, les contraintes et critères varient d'un type à l'autre mais, de manière plus significative, chacun des types ou sous-types remplit une fonction de PRÉCURSEUR vrai du type ou sous-type suivant.

Chacun des types ou sous-types de traductions répertoriés obéit à des contraintes spécifiques nées, chaque fois. de la nature des objectifs. Les critères de bonne traduction sont déterminés par les fonctions du texte traduit: fonctions de communication variant d'une situation à l'autre. Les critères de bonne traduction déterminent, à leur tour, les compétences nécessaires pour la maîtrise d'un type ou sous-type de traduction donné.

Pour clarifier les organisations ou progressions des démarches conduisant à la maîtrise des stratégies de la traduction correspondant aux divers types et sous-types possibles, nous retiendrons un schéma fondamental exigeant, dans cet ordre :

1. la définition stricte d'un PROJET DE TRADUCTION, incluant:

les déterminants de la traduction: public; destination ou fonctions; orientation; critères de qualité ou adéquation des transferts.

Le projet de traduction, toujours spécifique, constitue la donnée fondamentale gouvernant la totalité de l'acte. 
2. la formation (si nécessaire) de l'OBJET DE LA TRADUCTION.

L'objet de la traduction se définit comme:

totalité du texte anglais dans le cas de la traduction absolue;

para-texte dans certains cas de traductions (synoptiques ; diagrammatiques ;...)

Si la totalité du texte correspond "toujours» à l'unité de travail, certains types de traduction n'exigent pas le transfert de la totalité des données textuelles (informatives et stylistiques / rhétoriques). En pareil cas, il peut s'avérer nécessaire de mettre en place, en ANGLAIS, les éléments faisant effectivement l'objet d'un transfert JUSTIFIÉ.

3. la définition d'un TRAJET DE RÉALISATION DE LA TRADUCTION:

a. stratégies de la compréhension du texte anglais (avec vérification des résultats);

b. stratégies de la recherche documentaire;

c. stratégies du transfert;

d. stratégies de la rédaction et de la révision.

Les UNITÉS D'APPRENTISSAGE proposées s'organisent selon une progression raisonnée reposant sur le principe essentiel qui veut que la compréhension NÉCESSAIRE du texte soit assurée avant incidence de types de traductions successifs eux-mêmes enchâssés selon une progression des niveaux de compétence exigibles. Chaque unité d'apprentissage s'accompagne de travaux pratiques ou travaux dirigés regroupés en séries homogènes.

\section{NATURE ET ENJEUX DE L'ACTE DE TRADUCTION EN CONTEXTE «PROFESSIONNEL»}

Avant d'aborder les apprentissages relatifs à l'acte de traduction, il n'est sans doute pas inutile de s'interroger sur la nature et les enjeux de cet acte particulier.

L'acte de traduction se définit comme l'ensemble des activités résultant de la substitution d'au moins un déterminant de la production d'un message. D'un point de vue pratique, nous considérons qu'il y a traduction dès l'instant où la destination du message change. Cette destination est d'abord le public (ensemble de lecteurs connu ou visé); elle est - ensuite et corrélativement - l'exploitation ou utilisation visée du message.

Les déterminants de la production d'un message sont les déterminants du contexte interpersonnel (nature de l'auteur; nature du lecteur ; relations ou écarts entre l'un et l'autre dans les domaines culturels et / ou linguistiques), la nature de l'objet du texte (concept traité dans le texte), les finalités de traitement de l'objet du texte (finalités d'indexation, d'affect, d'exploitation, de communication) et, parfois - lorsque ceci est «imposé» - le type de texte.

Le cas typique (définitoire) de traduction est celui qu'entraîne la substitution du vecteur linguistique (langue) dans lequel est transmis le message. Il faut cependant ne pas perdre de vue que la traduction n'est pas simplement une conversation interlinguale (par substitution linguistique): la traduction peut également être phénomène intralingual (la langue de communication du message ne change pas). Dans ce dernier cas, le public ou la finalité d'exploitation change à l'intérieur d'une même communauté linguistique. Ceci explique que l'on puisse parler de traduction intralinguale lorsque, par exemple, on $\mathrm{vul}$ garise un texte spécialisé ou encore lorsque l'on modifie la tonalité d'un texte.

La finalité d'exploitation ou d'utilisation du texte doit être prévue par l'auteur avant que ne commence la rédaction. Cette finalité d' exploitation commande les fonctions du texte (fonctions utilitaires ou fonctions esthétiques).

L'acte de traduction professionnelle impliquera toujours une substitution linguistique. Il pourra parallèlement impliquer une modification naturelle des finalités du texte traduit par rapport au texte à traduire. Dans la série des apprentissages proposés, la modi- 
fication des finalités de communication permettra la maîtrise des procédures de traduction intralinguale ou, plus simplement, la prise en compte globale des déterminants de l'acte de traduction (ces déterminants construisant, en leur point de rencontre, le projet de traduction).

La modification naturelle des finalités du texte traduit est modification automatiquement induite par la substitution de destinataires.

L'acte de traduction suppose des critères de qualité qui ne peuvent pleinement se réduire à des critères d'équivalence. Nous parlerons de critères de congruence: lorsque l'on traduit un texte, il va de soi que l'équivalence est d'abord équivalence de messages - sauf si la modification des déterminants est telle que l'on doive modifier aussi les éléments de contenu ou leur forme. Mais l'équivalence de messages ne vaut réellement que pour la traduction absolue. Nous préférerons par conséquent substituer au critère d'équivalence un triple critère de congruence:

Le terme "congruence» a été choisi par extension du sens mathématique (se dit de deux nombres qui donnent le même résultat si on les divise par un même chiffre). Il désigne pour nous deux textes qui donnent un même "résultat" si on les renvoie à un même "projet textuel» compte tenu des divergences culturelles-expérientielles et linguistiques-rhétoriques qu' implique la substitution de communautés de lecteurs (franchissement de frontières culturelles et/ ou linguistiques).

a. Congruence absolue:

La congruence absolue s'analyse en référence aux normes en usage chez les lecteurs d'un texte: normes linguistiques (grammaire, orthographe, syntaxe) et normes culturelles (logique, perception et analyse des univers).

La congruence absolue est «impératif absolu» : la qualité d'une traduction c'est d'abord le fait qu'elle soit «lisible» (culturellement naturelle pour son lecteur aussi bien que linguistiquement «correcte»).

\section{b. Congruence-projet de traduction:}

Les critères d'évaluation de la traduction ne sont pas critères d'équivalence absolue au sens où il n'existerait, pour un texte donné, qu'un texte équivalent. Il faut juger le traducteur sur le respect ou le non-respect de son projet et, au second degré, juger son projet sur son adéquation ou inadéquation aux déterminants de l'acte de traduction. Ainsi, une traduction parfaite au sens académique peut être considérée comme nulle et non avenue si elle ne répond pas aux exigences de la situation créée entre le traducteur et son client. Sans aller si loin, le projet de traduction peut être tel que certains éléments du texte à traduire deviennent parasites s'ils sont conservés dans le texte français et, à l'inverse, les destinations peuvent être telles - pour le texte français - qu'elles contraignent à AJOUTER des informations que l'anglais renvoie à l'implicite.

Il n'existe pas davantage de "traduction UNIQUE d'un texte donné» qu'il n'existe de «manière unique» de construire un texte pour une information et un public donnés. Il est sans doute bon de savoir que nombre d'organismes recrutant des traducteurs imposent aux candidats des épreuves consistant en une série de variations à partir d'un texte donné: l'objectif est de mesurer la sensibilité des candidats aux variations de publics et de finalités des textes.

Les mauvaises traductions trouvent leur origine dans l'inadéquation des textes traduits à leur public et à leurs destinations ou utilisations explicites. 
Il importe donc:

a. que le projet de traduction soit adéquatement formé;

b. que le projet de traduction soit respecté.

Dans la pratique, la nature du projet de traduction peut et doit faire l'objet d' une négociation entre le traducteur et le «donneur d'ouvrage» ou demandeur de traduction.

c. Congruence informative et formelle de texte à texte:

Le troisième type de congruence intertextuelle est plus ténu. Il n'en demeure pas moins vrai que les deux textes (texte anglais à traduire puis texte français) doivent conserver une convergence réduite aux limites du projet de traduction. Le degré de congruence de texte à texte sera d'autant plus élevé que les déterminants de l'un et l'autre seront proches (compte tenu de l'écart définitoire induit par la confrontation de deux cultures-expériences et de deux langues-rhétoriques qui ne convergent absolument qu'en de rares circonstances).

Les équivalences de texte à texte sont d'autant plus précises et nombreuses que les publics de l'un et de l'autre sont eux-mêmes proches dans leur expérience (univers semblable) et leur culture (perception et interprétation semblables de leur univers).

Il faut surtout prendre conscience du fait que les critères d'équivalence ou, mieux, de congruence, varient selon la taille des unités de transfert considérées. Ainsi, on peut affirmer que tel mot de l'anglais et tel mot du français sont équivalents dans telles limites. On peut également affirmer que telle phrase de l'anglais et telle phrase du français sont proches ou équivalentes (surtout lorsque ces phrases correspondent l'une et l'autre à une stéréotypie de situation d'énonciation). Il devient plus difficile de parler d'équivalence de paragraphes et, plus encore, d'équivalences de textes complets. Nous envisagerons donc la rupture de congruence sous l'angle (et le vocable) de distorsions par rapport aux déterminants du projet de traduction - déterminants au nombre desquels figure en bonne place le texte à traduire servant de support à (et préexistant à) l'acte de traduction.

Ayant défini l'acte de traduction comme la résultante d'une substitution de déterminants de la production de textes puis posé trois types de congruence, il nous faut nous interroger sur les caractères vrais de la substitution.

De toute évidence, la substitution de déterminants est d'abord une substitution linguistique dans la mesure où l'on change de système de langue et d'usages: la grammaire et la rhétorique changent. Il s'agit là de la substitution visible (quoique pas toujours pleinement prise en compte en raison des attractions ou asservissements de rhétorique à rhétorique conduisant aux formes de calques linguistiques). En réalité, la substitution profonde (et cachée) est une substitution de systèmes culturels et d'univers expérientiels: on peut, en quelque sorte, affirmer que la grammaire culturelle change avant que ne change lla grammaire linguistique. Les univers infratextuels sont différents pour le texte anglais et le texte français puisque la sémantique (sens) se construit en référence aux algorithmes de la grammaire culturelle spécifique à la communauté des lecteurs du texte considéré.

Le danger majeur résiderait dans une définition simpliste de la traduction qui s'arrêterait à une modification de la forme linguistique implicitement réduite, elle-même, à un système de code indépendant des représentations qu'elle assure des algorithmes et unités de la grammaire culturelle.

L'univers infratextuel est l'univers dont le texte constitue une forme d'interprétation. Il préexiste à l'écriture mais peut se trouver modifié (interprété) lui-même par la production du texte. Il correspond à l'ensemble des expériences ou perceptions et aux algorithmes selon lesquels cet ensemble est traité dans une communauté (ou groupe) donnée. 
On trouve des exemples extrêmes d'effets de la substitution des grammaires culturelles et des modalités d'interprétation d'univers expérientiels différents dans la traduction des messages publicitaires ou, sur un autre plan, dans la traduction juridique confrontant deux codes différents. Les exemples extrêmes ne doivent pas masquer la réalité de la constance des écarts culturels-expérientiels qui ne s'amenuisent véritablement que par l'effet d'assimilations culturelles lorsque deux groupes appartenant à des communautés linguistiques différentes partagent des concepts identiques et des modalités identiques d'analyse, d'interprétation et de combinaison de ces concepts identiques.

L'assimilation culturelle forme des groupes interlinguistiques homogènes. Ainsi, dans des domaines spécifiques, on constate des similitudes de "grammaire culturelle» entre "spécialistes". La structuration de l' univers informatique de l'informaticien français n'est pas fondamentalement différente de celle de l'univers informatique de l'informaticien anglais. Il y a, dans ces limites, acculturation identique (ou presque).

Dans la traduction, il y a franchissement de frontières culturelles et franchissement de frontières linguistiques. Le franchissement de frontières linguistiques implique que l'on doive «dire autrement»; le franchissement de frontières culturelles-expérientielles implique que l'on doive aussi, fréquemment, «dire autre chose» ou, pour le moins, que ce que l'on dit n'est pas traité de la même manière que ce que disait le texte anglais: l'un et l'autre texte renvoient à des modalités différentes d'interprétation ou mise en relation avec un univers. Il importe donc d'éviter de produire un texte français que seuls pourraient véritablement "comprendre» des anglophones. Le texte anglais a été produit pour (en fonction de) son public anglo-saxon; le texte français doit être produit pour (en fonction de) son public français.

La traduction comporte nécessairement une part d'adaptation CULTURELLE qui est adaptation au public en même temps qu'aux destinations-utilisations du texte. La rémanence d' un écart culturel irréductible (même au moyen de la documentation) provoquera une rupture de compréhension et, donc, une impossibilité au moins partielle d'exploitation du texte: on ne peut rien "faire» d'un texte ou segment de texte que l'on ne comprend pas. On peut simplement faire des inférences risquées.

Il faut cependant nuancer : la confrontation de systèmes ne se réduit généralement pas dans une convergence que le traducteur instituerait en créant une situation d'équilibre. Dans l'acte de traduction, il existe une dominance culturelle-expérientielle. Cette dominance peut être accordée à la communauté à laquelle appartenait l'auteur du texte à traduire et, dans ce cas, la traduction a pour effet de montrer comment cette communauté voit et dit les choses. En d'autres termes, le traducteur ne se contente pas de communiquer l'information : il montre aussi à ses lecteurs comment fonctionne l'anglais. La traduction intègre alors une composante descriptive des caractères du texte anglais à traduire.

La fonction du traducteur est de créer un équilibre toujours précaire. Il trouve parfois des formes d'équilibre à caractère "absolu» (lorsque termes ou locutions convergent). Il ne peut cependant ignorer que les écarts culturels-expérientiels ne se comblent jamais totalement et qu'il devra le plus souvent trouver les compromis les «moins inacceptables».

Dans l'autre sens, la dominance peut être accordée au lecteur du texte traduit. Dans ce cas - cas le plus fréquent dans la traduction professionnelle - la traduction est processus d'assimilation culturelle-expérientielle et linguistique-rhétorique. L'assimilation a pour effet de rendre l'information (contenu du message) et sa forme (vecteur linguistique) totalement transparentes pour le public visé. Tout se passe alors comme si le traducteur, prenant appui sur le texte original, rédigeait «son» propre texte en fonction de son public 
et des finalités imposées ou souhaitées. La dominance est ainsi accordée aux systèmes de la communauté des lecteurs du texte traduit.

Il va de soi que l'orientation de la dominance constitue l'un des déterminants najeurs du projet de traduction dans la mesure où elle conditionne les qualités et quantités de congruences de texte à texte. Il faudra donc déterminer très soigneusement - lorsque le choix existe - l'orientation de la dominance et, par contrecoup, les modalités de confrontation des systèmes permettant de construire l'interprétation de chacun des textes.

La "rédaction" qu'entreprend le traducteur n'est pas, sauf cas exceptionnels, une rédaction «libre».

Nous parlerons par conséquent de "rédaction contrainte» par des impératifs de congruence intertextuelle. Si nous conservons la désignation de "rédaction", c'est pour mettre en évidence la nécessité, pour le traducteur, de conduire en parfaite connaissance de cause le déroulement de son texte.

En matière de traduction de textes pragmatiques, l'orientation est de type «assimilatoire» puisque l'abjectif est l'accès à une information-contenu et puisqu' il y a déclassement relatif des caractères stylistiques (sauf stéréotypies de formulations).

Lorsque - dans l'immense majorité des cas - la dominance est accordée aux systèmes et usages de la communauté des lecteurs du texte traduit, il importe de prendre en considération le contexte de jugement porté sur la traduction. En effet, contrairement à ce qui se passe en situation scolaire ou universitaire, l'utilisateur de la traduction ou le donneur d'ouvrage portant jugement sur la traduction risque, par définition, de ne pouvoir juger que sur des critères externes: critères de qualité de rédaction; critères de lisibilité; respect de la logique; précision ou justesse technique. Il n'intervient pas, dans le jugement porté, de référence au texte anglais à traduire et le texte français doit alors être pleinement transparent, autonome, autosuffisant. Il ne devra notamment comporter aucune faute de langue ou de style ou de présentation car on risque de s'exposer à des jugements sommaires de type: Ce ne peut être un bon traducteur puisqu'il ne connaît même pas sa langue!

Le donneur d'ouvrage ou client du traducteur ne peut, dans la majorité des cas, faire porter son évaluation que sur la qualité intrinsèque du texte français qui doit donc être «irréprochable».

Les relations entre traducteur et donneur d'ouvrage ou client ne s'apparentent en rien aux relations entre étudiant et professeur. La formation des traducteurs s'en trouve naturellement de plus en plus fréquemment confiée à des professionnels dont l'enseignement inclut une analyse des relations entre traducteurs et donneurs d'ouvrage.

L'apprentissage de la traduction professionnelle implique que les sujets soient soumis à des jugements "subjectifs» comparables à ceux que pourraient émettre les professionnels et, notamment, les «clients».

RÉCAPULATIF :

La traduction résulte d'une substitution de destinations induite par un franchissement de frontières linguistiques.

Le franchissement des frontières linguistiques est aussi et avant tout un franchissement de frontières culturelles-expérientielles.

Les critères de congruence sont triples: respect des normes absolues d'usage linguistique et culturel ; respect des déterminants du projet de traduction; respect des congruences intertextuelles.

Le projet de traduction doit inclure la préemption des modalités d'équilibre entre systèmes confrontés par-delà les frontières linguistiques et culturelles. 